\title{
Endoscopic surgery for nonsyndromic craniosynostosis: a 16-year single-center experience
}

\author{
Cecilia L. Dalle Ore, BA, ${ }^{1}$ Monisha Dilip, BS, ${ }^{1}$ Michael G. Brandel, BA, ${ }^{1}$ Joyce K. McIntyre, MD, ${ }^{2}$ \\ Reid Hoshide, MD, ${ }^{1}$ Mark Calayag, MD, ${ }^{3}$ Amanda A. Gosman, MD, ${ }^{2}$ Steven R. Cohen, MD, ${ }^{2}$ and \\ Hal S. Meltzer, MD ${ }^{1,3}$
}

1'Department of Neurosurgery, University of California San Diego; and Divisions of ${ }^{2}$ Plastic Surgery and ${ }^{3}$ Pediatric Neurosurgery, Rady Children's Hospital San Diego, California

OBJECTIVE In this paper the authors review their 16-year single-institution consecutive patient experience in the endoscopic treatment of nonsyndromic craniosynostosis with an emphasis on careful review of any associated treatmentrelated complications and methods of complication avoidance, including preoperative planning, intraoperative management, and postoperative care and follow-up.

METHODS A retrospective chart review was conducted on all patients undergoing endoscopic, minimally invasive surgery for nonsyndromic craniosynostosis at Rady Children's Hospital from 2000 to 2015. All patients were operated on by a single neurosurgeon in collaboration with two plastic and reconstructive surgeons as part of the institution's craniofacial team.

RESULTS Two hundred thirty-five patients underwent minimally invasive endoscopic surgery for nonsyndromic craniosynostosis from 2000 to 2015. The median age at surgery was 3.8 months. The median operative and anesthesia times were 55 and 105 minutes, respectively. The median estimated blood loss (EBL) was $25 \mathrm{ml}$ (median percentage EBL $4.2 \%$ ). There were no identified episodes of air embolism or operative deaths. One patient suffered an intraoperative sagittal sinus injury, 2 patients underwent intraoperative conversion of planned endoscopic to open procedures, 1 patient experienced a dural tear, and 1 patient had an immediate reexploration for a developing subgaleal hematoma. Two hundred twenty-five patients $(96 \%)$ were admitted directly to the standard surgical ward where the median length of stay was 1 day. Eight patients were admitted to the intensive care unit (ICU) postoperatively, 7 of whom had preexisting medical conditions that the team had identified preoperatively as necessitating a planned ICU admission. The 30-day readmission rate was $1.7 \%$ (4 patients), only 1 of whom had a diagnosis (surgical site infection) related to their initial admission. Average length of follow-up was 2.8 years (range $<1$ year to 13.4 years). Six children $(<3 \%)$ had subsequent open procedures for perceived suboptimal aesthetic results, 4 of whom (> 66\%) had either coronal or metopic craniosynostosis. No patient in this series either presented with or subsequently developed signs or symptoms of intracranial hypertension. CONCLUSIONS In this large single-center consecutive patient series in the endoscopic treatment of nonsyndromic craniosynostosis, significant complications were avoided, allowing for postoperative care for the vast majority of infants on a standard surgical ward. No deaths, catastrophic postoperative morbidity, or evidence of the development of symptomatic intracranial hypertension was observed.

https://thejns.org/doi/abs/10.3171/2018.2.PEDS17364

KEYWORDS craniosynostosis; endoscopic; minimally invasive; complications; metopic; coronal; sagittal; craniofacial

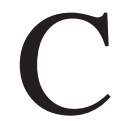
ONGENITALLY abnormal head shapes have been documented since ancient times. In 1851, Virchow described the pathophysiology of craniosynostosis, recognizing that "bony expansion ceases in a direc- tion perpendicular to synostosed suture, with compensatory expansion in the opposite direction." 35 Since the first known surgical repair of craniosynostosis was performed by Lannelongue in $1890,{ }^{4}$ numerous surgical techniques

ABBREVIATIONS CVR = cranial vault reconstruction; EBL = estimated blood loss; FOA = frontoorbital advancement; ICP = intracranial pressure; ICU = intensive care unit; $\mathrm{OR}=$ odds ratio; $\mathrm{PRBC}=$ packed red blood cell.

ACCOMPANYING EDITORIAL See pp 331-334. DOI: 10.3171/2018.3.PEDS18122.

SUBMITTED June 29, 2017. ACCEPTED February 21, 2018.

INCLUDE WHEN CITING Published online July 6, 2018; DOI: 10.3171/2018.2.PEDS17364. 
have been proposed utilizing long, typically "ear-to-ear" incisions and increasingly involved cranial vault and skull base techniques aimed at optimizing aesthetic and developmental outcomes. . $^{61,34}$

In contrast to these "open" approaches, minimally invasive or "endoscopic" craniosynostosis repair was first described by Vicari et al. in 1994. The authors introduced an endoscope during a sagittal craniosynostosis repair in an effort to minimize incision length while maintaining adequate anatomical visualization. ${ }^{43}$ In 1998, Jimenez and Barone reported their technique using endoscopic strip craniectomy combined with postoperative cranial orthotic molding. ${ }^{25}$

Since that time, numerous publications from multiple centers have described minimally invasive craniosynostosis repair with uniformly outstanding results and only rare reports of severe treatment-related complications. ${ }^{3,9}$, $23,27,32,36,37$ Recently, however, concerns have been raised regarding underreporting of suboptimal endoscopic treatment outcomes and anecdotal, unpublished reports of severe treatment-related complications, including massive intraoperative hemorrhage and death..$^{29} \mathrm{We}$ therefore present our large, consecutive patient, single-center experience over the last 16 years in the endoscopic treatment of craniosynostosis with an emphasis on careful examination of potential suboptimal treatment outcomes, as well as treatment-related complications and their avoidance.

\section{Methods}

A retrospective chart review was conducted of all patients undergoing primary endoscopic repair of craniosynostosis at Rady Children's Hospital San Diego between January 2000 and December 2015.

\section{Data Collection}

Following IRB approval, relevant data were extracted from patient charts. Data were obtained from anesthesia records, progress notes, discharge summaries, and operative notes. Preoperative and demographic data collected included date of birth, sex, weight, craniosynostosis diagnosis, American Association of Anesthesiologists classification, surgical duration, estimated blood loss (EBL), blood product administration, and intraoperative complications. Postoperative data were collected including postoperative disposition, length of stay, postoperative complications, postoperative transfusions, 30-day readmission, reoperations, and length of follow-up.

\section{Study Cohort}

Consecutive patients who underwent initial endoscopic repair of nonsyndromic craniosynostosis between January 2000 and December 2015 were included in the study. Patients with prior surgical repair of craniosynostosis or a syndromic diagnosis were excluded from the study. Diagnoses were confirmed by independent clinical examination supplemented with diagnostic CT in all cases. All procedures were performed by one neurosurgeon (H.S.M.) and one of two plastic surgeons (S.R.C., A.A.G.) as part of our institutional craniofacial team.

\section{Patient Selection and Operative Timing}

Endoscopic repair was typically offered as a treatment option for children with nonsyndromic craniosynostosis up to 6 months of age.

\section{Operative and Postoperative Management}

The perioperative protocol was consistent throughout the series. Patients received intravenous antibiotics, $0.5 \mathrm{~g} /$ $\mathrm{kg}$ mannitol, and $0.5 \mathrm{mg} / \mathrm{kg}$ dexamethasone prior to skin incision. Patients underwent intraoperative precordial Doppler ultrasound monitoring. Intraoperative pCO2 was targeted at $20-25 \mathrm{~mm} \mathrm{Hg}$. Patients had three postoperative hematocrit levels drawn over 24 hours following surgery. Intraoperative transfusion decisions were primarily made by the attending pediatric anesthesiologist. Postoperative transfusion decisions were made by the surgical team, with a typical hematocrit transfusion threshold of $21 \%$. Cranial orthotics were utilized postoperatively with the number and duration determined on an individual basis.

\section{Surgical Technique}

Following anesthesia induction, at least two intravenous lines and a bladder catheter were placed. The scalp was infused with $0.25 \%$ bupivacaine solution and needletip electrocautery was used to perform scalp incisions.

\section{Sagittal Synostosis}

Patients undergoing repair of sagittal synostosis were placed in a prone/modified sphinx position, as previously described. ${ }^{15}$ Three- to 4-centimeter incisions were made at the anterior and posterior fontanelles. Blunt dissection through the subgaleal space was followed by careful dissection of the dural edge at the posterior margin of the anterior fontanelle and the placement of a burr hole at the posterior aspect of the sagittal suture. The underlying dura and sagittal sinus were then dissected from the overlying fused sagittal suture, and the endoscope was introduced into the epidural space and used to assess for adequate dissection and ensure dural integrity. A sagittal suturectomy, lateral wedge osteotomies, and occipital osteotomies were performed to allow for intraoperative cranial vault remodeling.

\section{Metopic Craniosynostosis}

Patients undergoing repair of metopic synostosis were placed supine, and a horizontal skin incision was made over the anterior fontanelle region. In our initial experience, suturectomy was performed to the glabellar region. However, in an attempt to improve aesthetic outcomes, we developed a modified extended technique using tarsal incisions to address the associated skull base deformity as previously described (Fig. 1) ${ }^{28}$ Bilateral superior tarsal incisions were made to expose the supraorbital and lateral orbital rims. Additional frontolateral incisions were also utilized as needed. The subgaleal plane was dissected to expose the metopic suture, and the dural plane was accessed and dissected via a midline burr hole or through the anterior fontanelle down to the nasofrontal suture. Under endoscopic guidance, a metopic suturectomy was performed, along with bilateral frontoorbital osteotomies. 


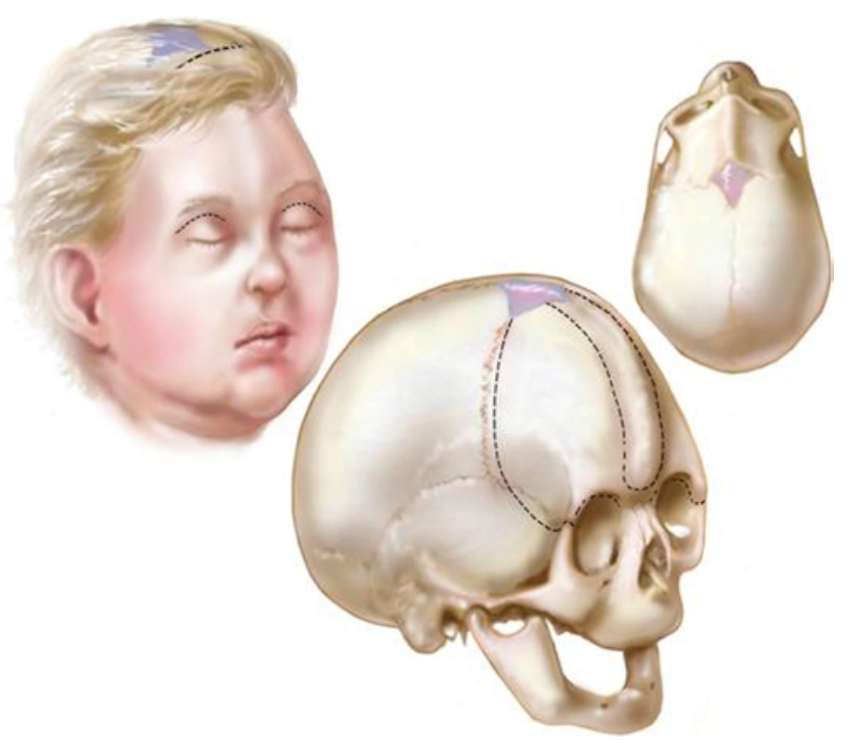

FIG. 1. Schematic illustration demonstrating the described endoscopic approach for metopic craniosynostosis. Dashed lines on the skin denote soft-tissue incisions and dashed lines on the skull illustrate osteotomies. Reproduced with permission from Keshavarzi S, Hayden MG, Ben-Haim S, Meltzer HS, Cohen SR, Levy ML: Variations of endoscopic and open repair of metopic craniosynostosis. J Craniofac Surg 20(5):1439-1444, 2009 (https://journals.Iww.com/jcraniofacialsurgery/Pages/default.aspx). Figure is available in color online only.

\section{Coronal Synostosis}

Initially, we performed coronal suturectomy lateral to the squamosal suture. However, as described above for metopic synostosis, we developed expanded techniques to correct coronal synostosis involving tarsal incisions and frontoorbital osteotomies in an attempt to improve our aesthetic outcomes by directly addressing the associated skull base deformity (Fig. 2). ${ }^{8}$

\section{Lambdoid Synostosis}

For lambdoid synostosis, a burr hole was placed in the affected midlambdoid region with suturectomy extended superomedially and inferolaterally along the fused lambdoid suture.

\section{Follow-Up}

All patients were followed postoperatively in the neurosurgery and craniofacial (plastic surgery) clinics by the respective attending physicians. Patients were also longitudinally followed by a neuroophthalmologist. Postoperative CT scans were not performed on a routine basis. Any clinical evidence of elevated intracranial pressure (ICP) - as indicated by headaches, visual changes, nausea, or behavioral changes - was investigated via imaging and ophthalmological assessment. Our protocol for invasive ICP monitoring has been previously published. ${ }^{1}$

\section{Patient Parameters}

Our patient parameters included death or significant morbidity (stroke, air embolism, significant intraoperative hemorrhage, seizure), readmission within 30 days, reop-

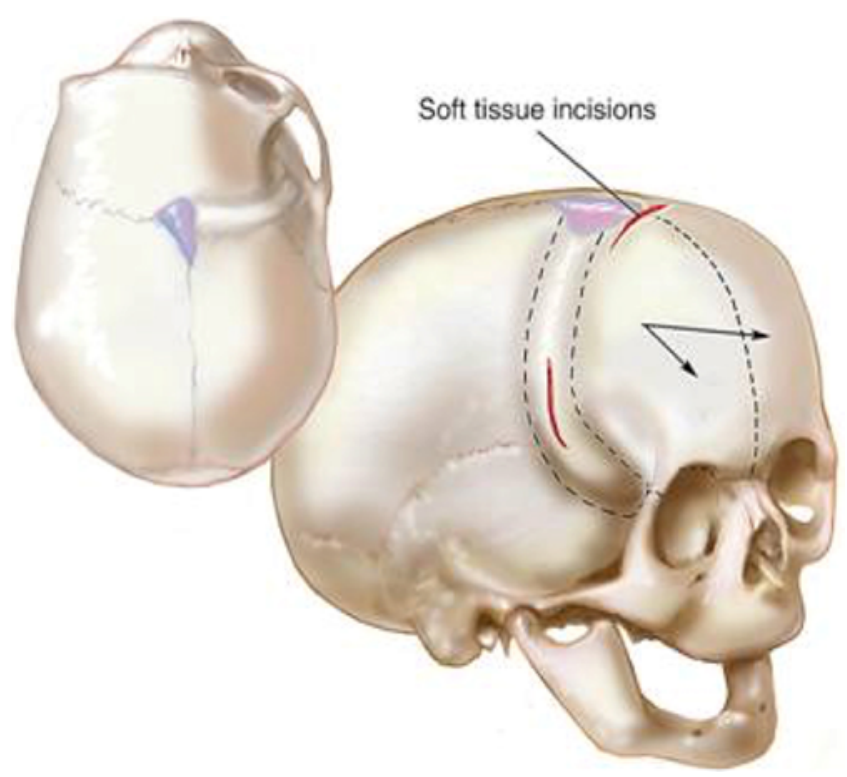

FIG. 2. Schematic demonstrating the described endoscopic approach for coronal craniosynostosis. Solid lines denote soft-tissue incisions, dashed lines denote osteotomies, and arrows indicate that the right frontal bone will be able to move forward to come into alignment with the left frontal bone. Reprinted from Clin Plast Surg 31(3), Cohen SR, Holmes RE, Meltzer HS, Nakaji P, Fronto-orbital and cranial osteotomies with resorbable fixation using an endoscopic approach, 429-442, Copyright 2004 , with permission from Elsevier. Figure is available in color online only.

eration, postoperative disposition, and other intraoperative and postoperative complications including any potential dural/venous sinus disruption, fevers, and transfusion reactions. We also identified operating room duration, EBL, administration of blood products, length of hospital stay, length of time in orthotic helmets, and length of follow up.

\section{Statistical Analysis}

The SPSS statistical program (version 24, IBM) and $\mathrm{R}$ (version 3.2.1, R Foundation for Statistical Computing) were used for statistical analysis. One-way ANOVA with a post-hoc Tukey analysis was used for the comparison of multiple groups. The threshold for statistical significance was set at $\mathrm{p}=0.05$. We utilized an a priori binary logistic regression model. Regression and analysis of operative parameters excluded the 2 patients who had intraoperative conversion from an initially planned endoscopic to an open procedure. For the purposes of our analysis, open reoperations were defined as frontoorbital advancements (FOAs)/cranial vault reconstructions (CVRs) that were performed jointly by a neurosurgeon and a craniofacial surgeon.

\section{Results}

Of the 235 patients who met inclusion criteria, $69 \%(\mathrm{n}=$ $162)$ of patients were male and $31 \%(n=73)$ were female. One hundred ninety patients $(81 \%)$ who underwent endoscopic repair presented with sagittal synostosis, 32 (14\%) 
TABLE 1. Demographics and operative parameters by diagnosis

\begin{tabular}{lcccccc}
\hline \multicolumn{1}{c}{ Variable } & Overall & Sagittal & Metopic & Coronal & Lambdoid & $p$ Value \\
\hline No. of patients (\%) & 235 & $190(80.8)$ & $32(13.6)$ & $11(4.7)$ & $2(0.8)$ & \\
\hline Age (mos) & 3.8 & 3.7 & 4.2 & 5.8 & 5.4 & 0.015 \\
\hline Weight (kg) & 6.7 & 6.7 & 6.8 & 6.7 & 6.9 & 0.557 \\
\hline EBL (ml) & 25 & 20 & 50 & 37.5 & 12.5 & $<0.001$ \\
\hline$\%$ EBL & 4.2 & 3.9 & 8.33 & 4.66 & 1.81 & $<0.001$ \\
\hline PRBCs (ml) & 62.1 & 52.5 & 117.7 & 77.2 & 0 & $<0.001$ \\
\hline$\%$ transfused & 46.7 & 43.7 & 67.7 & 50 & 0 & 0.065 \\
\hline Surgical duration (mins) & 55.5 & 55 & 59 & 96 & 19 & 0.057 \\
\hline Op room time (mins) & 105 & 105.5 & 103 & 86.5 & 99 & 0.865 \\
\hline Length of stay (days) & 1 & 1 & 2 & 1 & 1 & 0.987 \\
\hline
\end{tabular}

Values given as medians unless otherwise specified. One-way ANOVA was used to compare operative characteristics between craniosynostosis subtypes. Differences in EBL, PRBC administration, and age between diagnoses were statistically significant. In a post-hoc Tukey analysis, patients undergoing repair for sagittal craniosynostosis were significantly younger than coronal patients $(p=0.020)$ and patients with sagittal craniosynostosis had a significantly lower EBL $(p<0.001)$ and PRBC administration $(p<0.001)$ than those with metopic craniosynostosis.

* Mean calculated rather than median, as fewer than $50 \%$ of patients were transfused.

with metopic synostosis, $11(5 \%)$ with coronal synostosis, and $2(<1 \%)$ with lambdoid synostosis (Table 1). Two patients were converted intraoperatively to an open approach and were excluded from further analysis.

\section{Perioperative Data}

Patients had endoscopic repair at a median age of 3.8 months (range 1.05-12.7 months). The median surgical duration was 55.5 minutes (range 19-184 minutes). Median EBL was $25 \mathrm{ml}$ (range $<10$ to $350 \mathrm{ml}$ ) and median percentage EBL was $4.2 \%$ (range $<1 \%-41.3 \%$ ). One hundred eighteen patients $(50.2 \%)$ were transfused perioperatively. Of the transfused patients, $94(79.6 \%)$ were transfused solely intraoperatively, $9(7.6 \%)$ were transfused solely postoperatively, and $15(12.7 \%)$ were transfused both intraoperatively and postoperatively.

There was a statistically significant difference in ages between the diagnostic groups $(\mathrm{p}=0.015)$. Patients with sagittal craniosynostosis were significantly younger than patients with coronal craniosynostosis at time of repair ( $p$ $=0.020$ ).

The endoscopic repair of metopic craniosynostosis was associated with the highest median EBL at $50 \mathrm{ml}$ followed by coronal craniosynostosis with a median EBL of 37.5, sagittal craniosynostosis with a median EBL of $20 \mathrm{ml}$, and lambdoid with $12.5 \mathrm{ml}$. The difference in EBL between diagnostic groups was statistically significant $(\mathrm{p}<0.001)$, and patients with sagittal craniosynostosis had a significantly lower EBL than those with metopic craniosynostosis $(\mathrm{p}<0.001)$. Similarly, mean packed red blood cell (PRBC) administration was highest in metopic craniosynostosis at $117.7 \mathrm{ml}$, followed by coronal craniosynostosis $(77.2 \mathrm{ml})$, and sagittal craniosynostosis $(52.5$ $\mathrm{ml})$. The patients with lambdoid craniosynostosis did not undergo transfusion. The difference between the groups was statistically significant $(\mathrm{p}<0.001)$; patients with sagittal craniosynostosis received a lower volume of PRBCs than those with metopic craniosynostosis ( $\mathrm{p}<0.001)$. The transfusion rate was $43.7 \%$ in patients with sagittal cra- niosynostosis, $67.7 \%$ in patients with metopic craniosynostosis, $50 \%$ in patients with coronal craniosynostosis, and $0 \%$ in patients with lambdoid craniosynostosis $(\mathrm{p}=$ 0.065).

The endoscopic repair of coronal craniosynostosis had the longest median surgical duration at 96 minutes, followed by metopic at 59 minutes, sagittal at 55 minutes, and lambdoid craniosynostosis at 19 minutes. The difference between these groups trended toward statistical significance $(\mathrm{p}=0.057)$.

\section{Intraoperative Complications}

Two patients underwent intraoperative conversion from an endoscopic to an open approach (1 coronal, 1 metopic). The decision to convert to an open approach was based on the craniofacial surgeon's intraoperative perception of a likely suboptimal future aesthetic result. There was a statistically significant difference in rate of conversion to an open approach between the diagnoses (Table 2, p < 0.006 ), and the difference in rate of conversion to an open approach was significantly higher in coronal than sagittal craniosynostosis $(p=0.007)$. One patient undergoing sagittal craniosynostosis repair had a sagittal sinus injury managed with topical hemostatic agents. One patient undergoing repair for sagittal craniosynostosis was re-explored in the operating room immediately after closure when an enlarging subcutaneous fluid collection consistent with active subgaleal bleeding was noted as postoperative dressings were applied. One patient (with metopic craniosynostosis) was noted to have an intraoperative dural tear, which was managed by primary suture repair. No patient in this series developed a postoperative CSF leak, pseudomeningocele, or growing skull-fracture equivalent in follow-up.

\section{Postoperative Disposition and Complications}

Our standard protocol is to admit patients to a regular pediatric surgical ward after endoscopic craniosynostosis repair. Only 8 of 233 patients (3.4\%) who underwent endoscopic repair were admitted to the intensive care unit 
TABLE 2. Complications by diagnosis

\begin{tabular}{|c|c|c|c|c|c|c|}
\hline Diagnosis & Overall & Sagittal & Metopic & Coronal & Lambdoid & $p$ Value \\
\hline Conversion of endoscopic to open & $2(0.9 \%)$ & & $1(3.1 \%)$ & $1(0.9 \%)$ & & 0.006 \\
\hline Dural tear & $1(0.4 \%)$ & & $1(3.2 \%)$ & & & 0.089 \\
\hline Re-explored & $1(0.4 \%)$ & $1(0.5 \%)$ & & & & 0.974 \\
\hline Sagittal sinus injury & $1(0.4 \%)$ & $1(0.5 \%)$ & & & & 0.974 \\
\hline Readmission w/in 30 days & $4(1.7 \%)$ & $2(1.0 \%)$ & $2(6.3 \%)$ & & & 0.187 \\
\hline Reoperation & $7(3.0 \%)$ & $2(1.0 \%)$ & $3(9.7 \%)$ & $1(10 \%)$ & $1(50 \%)$ & $<0.001$ \\
\hline Debridement/hardware removal & $1(0.4 \%)$ & & & $1(10 \%)$ & & $<0.001$ \\
\hline Incision \& drainage & $1(0.4 \%)$ & & $1(3.2 \%)$ & & & 0.088 \\
\hline
\end{tabular}

One-way ANOVA was used to compare incidence of complications between the diagnoses. There was a statistically significant difference in reoperations between diagnostic groups, with significantly fewer reoperations in patients with sagittal craniosynostosis.

(ICU) after surgery. In 7 of these 8 patients, the ICU admission was planned for known preexisting significant comorbidities (congenital cardiac disease, coagulopathic history, etc.). The patient with the intraoperative sagittal sinus injury was observed in the ICU overnight. No patient required escalation of care from the regular surgical ward to the ICU. The median length of stay was 1 day (range 1-43 days) and there was no statistically significant difference in length of stay between the diagnostic groups.

The immediate postoperative complications noted were 1 patient with a self-limited fever (one-time measurement) and 1 patient with mild urticaria following blood transfusion. No deaths, air emboli, significant transfusion reactions, or postoperative seizures were noted.

\section{Readmissions}

Four patients (1.7\%) were readmitted within 30 days. One patient developed a postoperative wound infection with Staphylococcus aureus that was treated with operative irrigation and intravenous antibiotics. One patient was readmitted for observation of family-reported "wound drainage" and was discharged the following day without the need for any specific intervention. One patient was admitted for fever, nausea, and vomiting believed to be secondary to an unrelated viral upper respiratory tract infection. One patient was admitted overnight by the otolaryngology service for an unrelated otitis media with associated postauricular adenitis.

\section{Cranial Orthotics}

All patients had cranial orthotic therapy following surgical intervention. The median band time was 5.1 months (range 1-15.8 months), utilizing a median number of 2 bands (range 1-4 bands). One patient developed a superficial pressure sore and 1 patient developed contact dermatitis during orthotic therapy.

Cephalic indices (calculated by biparietal diameter/occipitofrontal diameter $\times 100$ ) were calculated for patients who underwent sagittal craniosynostosis repair (Table 3). The median preoperative cephalic index was 63.2 (range 46.2-79.7), median postoperative preband cephalic index was 67.3 (range 50.95-81.29), and median post-band cephalic index was 73.5 (range 63.9-87.43).

\section{Reoperations}

Fourteen patients $(6 \%)$ underwent reoperations, but only $7(3 \%)$ had a subsequent FOA or CVR: 6 for perceived suboptimal aesthetic outcomes, and 1 patient with initial sagittal craniosynostosis who then developed delayed unilateral coronal craniosynostosis. Of the 6 remaining FOA/ CVR patients, there were 3 metopic, 1 coronal, 1 lambdoid, and 1 sagittal. Therefore, only $1 \%$ of patients with sagittal synostosis had subsequent CVR, whereas $9.7 \%$ of patients with metopic craniosynostosis and $10 \%$ of coronal patients had subsequent CVR ( $<<0.001)$. These reoperations were performed at a median time of 12.3 months following endoscopic repair at a median age of 16.0 months.

The other reoperations consisted of 1 patient with a wound infection as discussed above, 2 patients (both with sagittal craniosynostosis) who had cranioplasty for residual skull defects, 1 coronal patient who required secondary scalp closure, and 3 patients (1 sagittal, 1 coronal, and 1 metopic) who had minimally invasive recontouring procedures without neurosurgical involvement.

\section{Follow-Up}

The average length of follow-up was 2.8 years (range $<1$ year to 13.4 years). No patient in this series developed clinically evident increased ICP or required invasive ICP monitoring.

\section{Multivariate Analysis}

The results of our multivariate, binary logistic regression for reoperation following endoscopic craniosynostosis repair are displayed in Table 4. After correcting for age

TABLE 3. Preoperative, postoperative, and post-banding cephalic indices

\begin{tabular}{cccc}
\hline Cephalic Index & Median & Min & Max \\
\hline Preop & 63.2 & 46.2 & 79.7 \\
\hline Postop & 67.3 & 50.9 & 81.3 \\
\hline Orthotic exit & 73.5 & 63.9 & 87.4 \\
\hline
\end{tabular}

Cephalic indices were calculated for patients with sagittal craniosynostosis. 
TABLE 4. Predictors of reoperation following endoscopic repair

\begin{tabular}{lccc}
\multicolumn{1}{c}{ Predictor } & B & p Value & OR \\
\hline Age (mos) & -0.177 & 0.504 & 0.838 \\
\hline$\%$ EBL & -1.078 & 0.887 & 0.34 \\
\hline Surgical duration (min) & -0.011 & 0.479 & 0.989 \\
\hline Sagittal diagnosis & -2.841 & $\mathbf{0 . 0 0 4}$ & 0.058 \\
\hline Transfused & 1.109 & 0.281 & 3.03 \\
\hline Band time (mos) & -0.091 & 0.612 & 0.913 \\
\hline Constant & -0.061 & 0.975 & 0.941 \\
\hline
\end{tabular}

The diagnosis of sagittal synostosis was associated with later reoperation when corrected for age, EBL, surgical duration, transfusion, and cranial orthotic time. Sagittal diagnosis was the sole statistically significant predictor of reoperation following endoscopic surgery (boldface type).

at time of repair, surgical duration and EBL, transfusion administration, and band time, diagnosis was the sole significant predictor of reoperation following endoscopic repair. There was a statistically significant reduction in the rate of reoperation for patients with sagittal craniosynostosis $(p=0.004$, odds ratio $[\mathrm{OR}]=0.058)$.

\section{Discussion}

Over the last two decades, endoscopic/minimally invasive techniques have emerged as viable treatment options for nonsyndromic craniosynostosis. 3,9,23,27,32,36,37 However, there have been recent published concerns regarding under- and nonreporting of significant treatment-associated complications. ${ }^{29}$

There is a potential risk of catastrophic hemorrhage and air embolism with craniofacial procedures. ${ }^{19}$ Three patients in this series (1.3\%) suffered significant bleeding events. In 1 of our patients with sagittal craniosynostosis, as the dura was being dissected from the edge of the posterior craniectomy, the outer wall of the sagittal sinus was inadvertently torn. Topical hemostatic agents readily controlled the bleeding and the endoscopic procedure was uneventfully completed. Another patient with sagittal craniosynostosis developed significant subgaleal bleeding during dressing placement. This was readily controlled with reapplication of bone wax. A third child with metopic craniosynostosis had persistent bleeding during removal of the frontal bone. Enlarging the skin incision or converting the procedure to an open approach might have facilitated application of bone wax and other topical agents to further limit intraoperative bleeding. ${ }^{15}$ As the incidence of venous air embolus may be lower, but still observed, in endoscopic versus open craniosynostosis procedures,,${ }^{14,40}$ we concur with those who advocate the prudent use of noninvasive intraoperative Doppler ultrasonography monitoring. ${ }^{11,37}$

Intraoperative dural tears may result in CSF leaks, pseudomeningoceles, and growing skull fracture equivalents after open and endoscopic craniosynostosis procedures. ${ }^{10,45}$ The patient in this series (with metopic craniosynostosis) that had an intraoperative dural tear noted and primarily repaired had an uneventful postoperative course.

In this series, almost $50 \%$ of the children were transfused either intra- or postoperatively. This likely reflects our relatively aggressive surgical approaches, a low threshold for transfusion administration by our anesthesia service, and our overall assessment of the risk/benefit analysis of transfusions. Our transfusion rate is therefore higher than that reported in other published endoscopic series. . $^{3,23,25,37}$ One of our patients developed posttransfusion urticaria, which was readily managed. None of the other 117 patients transfused had any complication noted referable to their receiving blood products. In San Diego County, donor blood is screened for hepatitis A, hepatitis $\mathrm{B}$, hepatitis C, and HIV. The risk of transmission of hepatitis C or HIV via blood transfusion is estimated to be lower than 1 in 1.3 million. ${ }^{30}$ When making transfusion decisions, we compare this theoretical risk to the risk of withholding blood products from a relatively low blood volume infant. Aggressive transfusion avoidance protocols using techniques such as immediate postoperative furosemide administration and/or allowance of very low postoperative hematocrit levels $\mathrm{s}^{5,20,23}$ may risk a potentially preventable catastrophic outcome. Historically, there were advocates for surgical protocols that incorporated "less complex" procedures or potential termination of craniofacial procedures should a transfusion be imminent. ${ }^{22}$ With modern blood banking techniques, the prioritization of transfusion prevention above other considerations is perplexing to us.

From a theoretical standpoint, minimally invasive approaches that avoid devascularized cranial autografts, foreign body implants, excessive brain retraction/manipulation, prolonged hospital/ICU stay, and prolonged operative/anesthetic times, should have limited associated complications. Only 1 patient $(0.4 \%)$ required an additional procedure for a postoperative infection (S. aureus), no patient experienced postoperative seizures, and 225 (96.5\%) of the patients were cared for on a regular surgical ward with a median overnight stay of 1 night. Seven of the 8 patients admitted to the ICU postoperatively were planned admissions for severe medical comorbidities. This included the patient with a prolonged (43-day) hospitalization who was a medically fragile inpatient at the time of surgery. No patient directly admitted to the surgical ward required transfer to a higher level of care. We believe this reflects careful preoperative evaluations performed in our craniofacial outpatient clinic. Similarly, we advocate for frequent postoperative clinic visits supplemented by intensive family education to help limit readmission rates. We find the use of midlevel health care professionals (nurse practitioners and/or physician assistants) to be an invaluable resource. With our aggressive outpatient treatment paradigm, we were able to limit our 30-day readmission rate to $1.7 \%$.

In general, our institutional philosophy has been to consider optimal aesthetic outcome to be the primary therapeutic goal in the treatment of nonsyndromic craniosynostosis, and our surgical techniques and treatment preferences have evolved over time based on this consideration. In this framework, we consider suboptimal aesthetic results to be a potential complication of craniosynostosis surgery. Our treatment experience has therefore led us to utilize more aggressive techniques in the endoscopic management of craniosynostosis at the expense of increased surgical time and transfusion requirement. We favor, for 
example, the addition of perpendicular bilateral anterior and posterior parietal osteotomies ("barrel stave" osteotomies) to the removal of the pathologic fused suture in the endoscopic treatment of sagittal craniosynostosis. We have been satisfied with our outcomes in sagittal synostosis using these enhanced endoscopic techniques. However, other groups primarily utilizing cephalic indices as an outcome measure have not had this same experience, resulting in advocacy for increasingly minimal surgical interventions (i.e., suturectomy alone) ${ }^{9,37}$ In this study we found a median improvement in cephalic index from 63.2 preoperatively to 67.3 immediately postoperatively and 73.5 after the completion of cranial banding. Our median preoperative cephalic index is lower than that reported in many other series, reflecting the severity of disease addressed in this series as well as the relatively advanced median age (3.7 months), while our postoperative cephalic index is well within the range of that achieved in other series. $3,9,27,34,36,37,41$ However, while cephalic index is a useful 2D objective data point, we believe it does not reflect the entirety of potential aesthetic issues observed in these children, such as frontal bossing and occipital tapering, that reflect the complex 3D nature of this deformity. The development of metrics that better capture the 3D nature of craniosynostosis and additional studies aimed at specifically capturing and quantifying aesthetic outcomes will hopefully allow for more rigorous comparison of different surgical techniques in the future.

Similarly, with regard to metopic and coronal synostosis, we were not satisfied with the outcomes we observed at this institution via strip craniectomy alone, and developed more aggressive surgical techniques to directly address the skull base deformities inherent to these diagnoses. ${ }^{7}$ Therefore, our operative times and EBLs were higher in patients with these diagnoses, although this did not translate to an increased transfusion incidence. However, even utilizing these direct skull base, minimally invasive approaches, we were ultimately still not satisfied with our aesthetic outcomes for patients with coronal and metopic synostosis. In the absence of specific, universal, granular, and objective 3D metrics of aesthetic outcomes, reoperation rates can serve as an indirect outcome measure. ${ }^{44}$ While only $3 \%$ of our patients overall had subsequent open repair (FOA/ CVR) following endoscopic surgery, there was a statistically significant increase in the rate of open reoperation for patients with initial coronal $(30 \%)$ and metopic (16\%) endoscopic repair compared to patients with sagittal repair $(2.8 \%)$. The lower rate of open reoperation in sagittal patients remained significant after correcting for age, EBL, surgical duration, and band time in a multivariate analysis. We typically now will consider endoscopic procedures only for infants with coronal or metopic craniosynostosis whose families are extremely motivated for an endoscopic procedure to be considered. This solely reflects evolution in our center's approach. We recognize that many reports from multiple other centers describe excellent results, including outstanding aesthetic outcomes and facial symmetry with a low rate of reoperation following endoscopic repair of coronal and metopic craniosynostosis, and that our reoperation rates for these diagnoses are not dissimilar to those described in children undergoing initial open approaches. ${ }^{12,16,17,24,31,33,38}$ Minimally invasive craniosynostosis procedures typically are supplemented by the temporary insertion of expansion devices such as cranial springs or, as in this series, the use of postoperative cranial molding orthotic devices., ${ }^{3,26,36}$ Expansion devices require a mandatory, typically very limited, secondary procedure under anesthesia to remove the implant. Recommendations have been published suggesting, if possible, limiting anesthetic exposures in this age group $\cdot{ }^{13}$ Alternatively, some authors have expressed concerns regarding cranial banding, specifically the financial and quality of life implications of a reported 12-18-month cranial orthotic regimen that we note is far longer than the 5.1-month median orthotic therapy duration in this series. ${ }^{18}$ We did identify 2 patients with complications specifically related to cranial banding. One child with metopic craniosynostosis developed a superficial pressure sore that resulted in modification of the cranial band treatment. This patient ultimately underwent a secondary open FOA. Another patient with sagittal craniosynostosis developed a severe contact dermatitis also requiring modification of the cranial band treatment. While not requiring a secondary open FOA to date, we continue to monitor this patient's overall aesthetic outcome. As noted in a study specifically of children using cranial orthotic devices to treat positional plagiocephaly, cranial banding does not automatically and universally result in an improved aesthetic appearance. ${ }^{42} \mathrm{We}$ believe that direct involvement by the surgical team in the cranial banding process as well as the availability of an experienced orthotic vendor are essential to achieving acceptable outcomes when utilizing a cranial band following minimally invasive craniosynostosis procedures.

It is estimated that up to $5 \%$ of children with nonsyndromic craniosynostosis may develop symptomatic intracranial hypertension. ${ }^{39}$ During follow-up visits, we carefully review any concerns related to chronic headaches or visual changes. Additionally, we advocate longitudinal neuroophthalmological care to assess for occult papilledema. We have previously published our protocol for the use of invasive ICP monitoring to aid in clinical decision-making for ICP-related craniofacial procedures. ${ }^{1}$ None of the patients in this series developed any clinical concerns for increased ICP or underwent invasive ICP monitoring. Recently, there have been published reports of more subtle developmental differences in children who underwent minimally invasive versus more traditional open procedures for the treatment of nonsyndromic craniosynostosis. ${ }^{21}$ It has been proposed that observed cognitive outcomes may be secondary to non-ICP related neurological abnormalities in this patient population. ${ }^{2}$ Prospective clinical trials will be needed to ultimately address these intriguing concerns regarding neurocognitive outcomes in patients with nonsyndromic craniosynostosis.

\section{Conclusions}

In this large single-center, single-neurosurgeon, consecutive patient series in the endoscopic treatment of nonsyndromic craniosynostosis, significant complications were avoided, allowing for postoperative care for the vast majority of infants on a standard surgical ward. No deaths, 
catastrophic postoperative morbidity, or evidence of the development of symptomatic intracranial hypertension was observed. Endoscopic procedures represent safe and efficacious options in the treatment of craniosynostosis.

\section{References}

1. Baird LC, Gonda D, Cohen SR, Evers LH, LeFloch N, Levy ML, et al: Craniofacial reconstruction as a treatment for elevated intracranial pressure. Childs Nerv Syst 28:411-418, 2012

2. Beckett JS, Brooks ED, Lacadie C, Vander Wyk B, Jou RJ, Steinbacher DM, et al: Altered brain connectivity in sagittal craniosynostosis. J Neurosurg Pediatr 13:690-698, 2014

3. Berry-Candelario J, Ridgway EB, Grondin RT, Rogers GF, Proctor MR: Endoscope-assisted strip craniectomy and postoperative helmet therapy for treatment of craniosynostosis. Neurosurg Focus 31(2):E5, 2011

4. Bir SC, Ambekar S, Notarianni C, Nanda A: Odilon Marc Lannelongue (1840-1911) and strip craniectomy for craniosynostosis. Neurosurg Focus 36(4):E16, 2014

5. Bonfield CM, Sharma J, Cochrane DD, Singhal A, Steinbok $\mathrm{P}$ : Minimizing blood transfusions in the surgical correction of craniosynostosis: a 10-year single-center experience. Childs Nerv Syst 32:143-151, 2016

6. Clayman MA, Murad GJ, Steele MH, Seagle MB, Pincus DW: History of craniosynostosis surgery and the evolution of minimally invasive endoscopic techniques: the University of Florida experience. Ann Plast Surg 58:285-287, 2007

7. Cohen SR, Holmes RE, Meltzer HS, Nakaji P: Immediate cranial vault reconstruction with bioresorbable plates following endoscopically assisted sagittal synostectomy. J Craniofac Surg 13:578-584, 2002

8. Cohen SR, Mittermiller PA, Meltzer HS, Levy ML, Broder KW, Ozgur BM: Nonsyndromic craniosynostosis: current treatment options, in Thaller SR, Bradley JP, Garri JI (eds): Craniofacial Surgery. New York: Informa Healthcare, 2008, p 95

9. Dlouhy BJ, Nguyen DC, Patel KB, Hoben GM, Skolnick GB, Naidoo SD, et al: Endoscope-assisted management of sagittal synostosis: wide vertex suturectomy and barrel stave osteotomies versus narrow vertex suturectomy. J Neurosurg Pediatr 25:674-678, 2016

10. Esparza J, Hinojosa J: Complications in the surgical treatment of craniosynostosis and craniofacial syndromes: apropos of 306 transcranial procedures. Childs Nerv Syst 24:1421-1430, 2008

11. Faberowski LW, Black S, Mickle JP: Incidence of venous air embolism during craniectomy for craniosynostosis repair. Anesthesiology 92:20-23, 2000

12. Farber SJ, Nguyen DC, Skolnick GB, Naidoo SD, Smyth MD, Patel KB: Anthropometric outcome measures in patients with metopic craniosynostosis. J Craniofac Surg 28:713-716, 2017

13. FDA: General anesthetic and sedation drugs: drug safety communication-new warnings for young children and pregnant women, in: Safety Alerts for Human Medical Products. Silver Spring, MD: FDA, 2016

14. Felema GG, Bryskin RB, Heger IM, Saswata R: Venous air embolism from Tisseel use during endoscopic cranial vault remodeling for craniosynostosis repair: a case report. Paediatr Anaesth 23:754-756, 2013

15. Frank R, Cohen SR, Meltzer HS: Craniosynostosis: frontoorbital advancement and cranial vault reshaping (open and endoscopic), in Jandial R, McCormick P, Black PM (eds): Core Techniques in Operative Neurosurgery. Philadelphia: Elsevier Saunders, 2011, Vol 1, pp 349-355

16. Gociman B, Agko M, Blagg R, Garlick J, Kestle JR, Siddiqi F: Endoscopic-assisted correction of metopic synostosis. J Craniofac Surg 24:763-768, 2013
17. Gociman B, Blagg R, Agko M, Goodwin I, Kestle JR, Siddiqi F: The metopic angle: a novel assessment tool of the trigonocephalic frontal deformity and its correction. J Craniofac Surg 25:2101-2104, 2014

18. Goodrich JT: Editorial. Cranial orthosis therapy. J Neurosurg Pediatr 12:203-205, 2013

19. Harris MM, Strafford MA, Rowe RW, Sanders SP, Winston KR, Rockoff MA: Venous air embolism and cardiac arrest during craniectomy in a supine infant. Anesthesiology 65:547-550, 1986

20. Harroud A, Weil AG, Turgeon J, Mercier C, Crevier L: Association of postoperative furosemide use with a reduced blood transfusion rate in sagittal craniosynostosis surgery. J Neurosurg Pediatr 17:34-40, 2016

21. Hashim PW, Patel A, Yang JF, Travieso R, Terner J, Losee JE, et al: The effects of whole-vault cranioplasty versus strip craniectomy on long-term neuropsychological outcomes in sagittal craniosynostosis. Plast Reconstr Surg 134:491-501, 2014

22. Hentschel S, Steinbok P, Cochrane DD, Kestle J: Reduction of transfusion rates in the surgical correction of sagittal synostosis. J Neurosurg 97:503-509, 2002

23. Honeycutt JH: Endoscopic-assisted craniosynostosis surgery. Semin Plast Surg 28:144-149, 2014

24. Jimenez DF, Barone CM: Early treatment of coronal synostosis with endoscopy-assisted craniectomy and postoperative cranial orthosis therapy: 16-year experience. J Neurosurg Pediatr 12:207-219, 2013

25. Jimenez DF, Barone CM: Endoscopic craniectomy for early surgical correction of sagittal craniosynostosis. J Neurosurg 88:77-81, 1998

26. Jimenez DF, Barone CM, Cartwright CC, Baker L: Early management of craniosynostosis using endoscopic-assisted strip craniectomies and cranial orthotic molding therapy. Pediatrics 110:97-104, 2002

27. Jimenez DF, Barone CM, McGee ME, Cartwright CC, Baker CL: Endoscopy-assisted wide-vertex craniectomy, barrel stave osteotomies, and postoperative helmet molding therapy in the management of sagittal suture craniosynostosis. J Neurosurg 100 (5 Suppl Pediatrics):407-417, 2004

28. Keshavarzi S, Hayden MG, Ben-Haim S, Meltzer HS, Cohen SR, Levy ML: Variations of endoscopic and open repair of metopic craniosynostosis. J Craniofac Surg 20:1439-1444, 2009

29. Kung TA, Vercler CJ, Muraszko KM, Buchman SR: Endoscopic strip craniectomy for craniosynostosis: do we really understand the indications, outcomes, and risks? J Craniofac Surg 27:293-298, 2016

30. Lavoie J: Blood transfusion risks and alternative strategies in pediatric patients. Paediatr Anaesth 21:14-24, 2011

31. MacKinnon S, Proctor MR, Rogers GF, Meara JG, Whitecross S, Dagi LR: Improving ophthalmic outcomes in children with unilateral coronal synostosis by treatment with endoscopic strip craniectomy and helmet therapy rather than fronto-orbital advancement. J AAPOS 17:259-265, 2013

32. Murad GJ, Clayman M, Seagle MB, White S, Perkins LA, Pincus DW: Endoscopic-assisted repair of craniosynostosis. Neurosurg Focus 19(6):E6, 2005

33. Nguyen DC, Patel KB, Skolnick GB, Naidoo SD, Huang AH, Smyth MD, et al: Are endoscopic and open treatments of metopic synostosis equivalent in treating trigonocephaly and hypotelorism? J Craniofac Surg 26:129-134, 2015

34. Panchal J, Marsh JL, Park TS, Kaufman B, Pilgram T, Huang SH: Sagittal craniosynostosis outcome assessment for two methods and timings of intervention. Plast Reconstr Surg 103:1574-1584, 1999

35. Persing JA, Jane JA, Shaffrey M: Virchow and the pathogenesis of craniosynostosis: a translation of his original work. Plast Reconstr Surg 83:738-742, 1989 
36. Ridgway EB, Berry-Candelario J, Grondin RT, Rogers GF, Proctor MR: The management of sagittal synostosis using endoscopic suturectomy and postoperative helmet therapy. J Neurosurg Pediatr 7:620-626, 2011

37. Shah MN, Kane AA, Petersen JD, Woo AS, Naidoo SD, Smyth MD: Endoscopically assisted versus open repair of sagittal craniosynostosis: the St. Louis Children's Hospital experience. J Neurosurg Pediatr 8:165-170, 2011

38. Tan SP, Proctor MR, Mulliken JB, Rogers GF: Early frontofacial symmetry after correction of unilateral coronal synostosis: frontoorbital advancement vs endoscopic strip craniectomy and helmet therapy. J Craniofac Surg 24:1190-1194, 2013

39. Thomas GP, Johnson D, Byren JC, Judge AD, Jayamohan J, Magdum SA, et al: The incidence of raised intracranial pressure in nonsyndromic sagittal craniosynostosis following primary surgery. J Neurosurg Pediatr 15:350-360, 2015

40. Tobias JD, Johnson JO, Jimenez DF, Barone CM, McBride DS Jr: Venous air embolism during endoscopic strip craniectomy for repair of craniosynostosis in infants. Anesthesiology 95:340-342, 2001

41. Utria AF, Lopez J, Cho RS, Mundinger GS, Jallo GI, Ahn ES, et al: Timing of cranial vault remodeling in nonsyndromic craniosynostosis: a single-institution 30-year experience. J Neurosurg Pediatr 18:629-634, 2016

42. van Wijk RM, van Vlimmeren LA, Groothuis-Oudshoorn CG, Van der Ploeg CP, Ijzerman MJ, Boere-Boonekamp MM: Helmet therapy in infants with positional skull deformation: randomised controlled trial. BMJ 348:g2741, 2014

43. Vicari F: Endoscopic correction of sagittal craniosynostosis, in Meeting of American Society of Plastic Surgeons. San Diego: ASPS, 1994

44. Williams JK, Cohen SR, Burstein FD, Hudgins R, Boydston W, Simms C: A longitudinal, statistical study of reoperation rates in craniosynostosis. Plast Reconstr Surg 100:305-310, 1997
45. Yamamoto M, Moore MH, Hanieh A: Growing skull fracture after cranial vault reshaping in infancy. J Craniofac Surg 9:73-75, 1998

\section{Disclosures}

The authors report no conflict of interest concerning the materials or methods used in this study or the findings specified in this paper.

\section{Author Contributions}

Conception and design: Meltzer, McIntyre, Cohen. Acquisition of data: Meltzer, Dalle Ore, Dilip, McIntyre. Analysis and interpretation of data: Meltzer, Dalle Ore, Dilip, Brandel, McIntyre, Hoshide, Calayag, Gosman. Drafting the article: Meltzer, Dalle Ore, Brandel, Hoshide, Gosman. Critically revising the article: Meltzer, Dalle Ore, Brandel, Hoshide, Gosman, Cohen. Reviewed submitted version of manuscript: all authors. Approved the final version of the manuscript on behalf of all authors: Meltzer. Statistical analysis: Dalle Ore, Dilip, Brandel, Hoshide. Study supervision: Meltzer, Cohen, Gosman.

\section{Supplemental Information}

\section{Previous Presentations}

Previously presented at the 84th AANS Annual Scientific Meeting, Chicago, Illinois, April 30 to May 4, 2016.

\section{Correspondence}

Hal S. Meltzer: Rady Children's Hospital San Diego, CA. hsmeltzer@ucsd.edu. 